\title{
Transgenic Animal - Mini Review
}

\section{Titas Sarkar*, Anamika Saha, Mayukh Maji, Dipak Kumar Singha and Manas Chakraborty}

Department of Pharmaceutical Biotechnology, Calcutta Institute of

Pharmaceutical Technology and AHS, Howrah, West Bengal, India

*Corresponding Author: Titas Sarkar, Department of Pharmaceutical

Biotechnology, Calcutta Institute of Pharmaceutical Technology and AHS, Howrah,

West Bengal, India.
Received: December 07, 2021

Published: January 10, 2021

(C) All rights are reserved by Titas Sarkar., et al.

\section{Abstract}

The word Transgenic stands for one or more DNA sequences from another species have been introduced by artificial ways. Transgenic animals are used as biomedical models in laboratory research, like rodent species. Rodent species which are important tools for researching human disease. These transgenic animals easily understands gene function in case of any disease and helps to determine the disease. Transgenic animals helps to produce complex human proteins in very large quantities. Transgenic animals have foreign genes in their genome and for this reason they can show some special properties other than normal animals. These properties lead to discover new drugs, helps in gene therapy etc. In case of gene therapy as defected genes could not function, so healthy genes are inserted by the help of vectors in th human body. Other than this, transgenic animals help in xerotransplantation. Pig is the experimental animal in case of xerotransplantation. Transgenic animals also play important role in agricultural field. Amount of milk with good quality and healthy meat have increased and farmers get benefited due to transgenic animals. These genetically modified animals are disease resistant and used in industrial purposes also.

Keywords: Embryonic Stem Cell; Mediated Transgenesis; Pronuclear Micro-injection; Somatic Cell Nuclear Transfer; Sperm Mediated Transgenesis; Transgenic Animals

\section{Introduction}

Transgenic animals carries a foreign gene that is deliberately instead into its genetic materials. It can be said that transgenesis is a process of production of hybridized animals by mixing up their genes taken from different sources of animals belonging to same or different ancestors. The first transgenic animal was a super-mouse developed in the year 1974 by Rudolf Jaenisch by administering foreign DNA into it's embryo. This was developed by inserting $\mathrm{Hu}-$ man Growth Hormone (HGH) into it. The progeny thus produced was larger in size compared to the parent. Transgenesis is being used in a wide range of field including the development of fishes, livestock and other animals to make it useful to mankind. Development of transgenic animals has been useful to study normal physi- ology and development including gene regulation, their effect on normal function of the body, study of disease by studying about the genes that are responsible for causing disease in human, also these animals helps in production of biological products with better efficiency and enhanced quality. These animals are development for safety test of vaccine, before applying on human.

\section{Regulation of gene expression}

Gene regulation has been examined extensively in vitro by expressing foreign gene constructs in cultured cells. However, the relevance of many cell culture studies to actual in vivo events is tenuous. Transgenic animals provide a true in vivo environment for evaluating the mechanisms by which gene expression is modulated in the adult and during development. Detailed analysis of myriad 
gene regulatory elements (promoters and enhancers) in the context of the whole animal has led to remarkable discoveries about gene function, and also to the development of more sophisticated tools for targeting the expression of heterologous genes to specific types of cells. For example, regulatory elements that control the complex tissue- and stage-specific pattern of a-fetoprotein expression during mouse embryogenesis have been identified by reproducing their activity in transgenic mice. Transgenic mice have also been used to identify regulatory control elements that are localized a great distance from the coding region of the gene. In the human 13-globin locus distant control elements, called locus control regions (LCR) or locus activation regions, are responsible for maximum appropriate expression in transgenic mice. In the absence of specific DNA se I hypersensitive sites in the LCR region, /3-globin expression is reduced approximately 300 -fold in transgenic mice. These regions may serve as nuclear matrix attachment sites, as binding sites for The integration of foreign DNA into the genome can perturb endogenous gene structure and function. Loss-of function mutations are usually recessive, requiring the insertional inactivation of both alleles. The discovery and characterization of insertional mutations caused by the integration of transgenes and the subsequent isolation and sequencing of the disrupted endogenous gene have led to advancements in our understanding of development and genetic disease. Because insertion of retroviruses and microinjected DNA sequences is generally random, specific mutations are not chosen, but are instead generated by chance. Nevertheless, these techniques have been used to create dozens of recessive mutations, several of which map to previously characterized loci such as down less, limb deformity, and hotfoot. If a mutant phenotype could be selected in vitro, randomly generated mutations could be more efficiently used. Mutated ES cells have been selected for the presence or absence of a functional hypoxanthine-guanine phosphoribosyl transferase (HPRT) gene before being used to generate a transgenic mouse model for Lesch-Nyhan syndrome. Because the HPRT gene is located on the X chromosome, a single intragenic mutation can completely inactivate HPRT function in male-derived ES cells, resulting in a readily detectable phenotype. Unfortunately, most genes are neither X-linked nor selectable. Targeted knockout of non selectable genes was achieved only by the development of technology permitting the generation and detection of rare sitespecific homologous recombination events in ES cells, including positive-negative selection and polymerase chain reaction. The parameters that govern the success of this technique have been thor- oughly described elsewhere. Genetically altered ES cells have been reintroduced into mouse embryos and allowed to colonize the germ line, causing heritable loss-of-function mutations. When homozygous transgenic mice are generated, the resulting phenotype demonstrates the function of the crippled endogenous gene. This approach has been used to achieve null mutations of the 132-microglobulin gene, resulting in the loss of major histocompatibility complex class 1 antigen and CD48 T cell-mediated cytotoxicity; the Wnt-1 protooncogene, resulting in the abnormal development of midbrain and cerebellum; the src protooncogene, resulting in osteopetrosis; and the Hox-1.5 gene, resulting in a constellation of phenotypic defects including abnormalities of the thyroid, parathyroid, thymus, salivary gland, heart, and the cartilage, bone, and musculature associated with the topoisomerase II or as erythroidspecific enhancers of replication. A serious problem in achieving efficient and predictable transgene expression is the potentially deleterious effect of endogenous genomic sequences near the site of integration. The presence of LCRs within transgenes may overcome the influence of these flanking sequences. Specific regions of mammalian DNA, A-elements, have been identified which mediate attachment of the chromatin to the nuclear scaffold. Reporter genes flanked by A-elements exhibit elevated expression that is insertion site-independent in stably transfected cultured cells. The availability of control elements that would render a transgene free from the unpredictable influences of the mouse genome would enable copy number dependent expression, thereby establishing a mechanism to achieve reproducibly higher levels of transgene transcription $[1,3,4]$.

Transgenic approach has provided new insight into cardiovascular homeostasis and disease. Overexpression of the mouse ren2 transgene in the rat adrenal gland elicits severe hypertension showed that production of rat renin together with angiotensinogen causes elevated systolic blood pressure in transgenic mice, which can be reversed by the angiotensin converting enzyme inhibitor captopril. In contrast, transgenic mice expressing the atrial natriuretic factor in the liver exhibit hypotension. The role of oncogenes in tumorigenesis is being elucidated by targeting the expression of various types of oncogenes to specific tissues of transgenic animals. The dominant expression of activated oncogenes often results in the development of heritable tumors, clearly implicating these genes in onco- genesis. Several cogent and informative reviews have been written on this subject. I will focus on a few sig- 
nificant aspects of oncogenes in transgenic mice. It has been recognized for many years that specific chromosomal translocations are associated with distinct forms of cancer. Molecular analysis of the chromosomal breakpoints have established that protooncogenes are often relocated and mutated. These rearrangement events can be recreated in vivo by generating transgenic mice possessing similarly modified genes. Transgenic mice bearing a deregulated fusion minigene consisting of the bcl-2 protooncogene juxtaposed to the immunoglobulin heavy-chain gene exhibit follicular lymphoid hyperplasia that can progress to lymphoma. Mice transgenic for a bcr-abl fusion gene [Philadelphia chromosome] demonstrate acute leukemia [2]. These kinds of transgenic experiments validate the causal relationship between translocations and their associated cancers. Transgenic animals afford the opportunity to test the hypothesis that cancer progresses in a multistep fashion, re- quiring activation of a number of oncogenes. Transgenic mice bearing distinct oncogenes are derived independently and then cross-mated to generate doubly transgenic animals. The synergistic behavior of ras and myc has been demonstrated in mammary gland differentiation and tumorigenesis. Cooperativity has also been established between ras, myc, and SV4O T-antigen in hepatocarcinogenesis $[1,5]$.

Transgenic animals afford the opportunity to test the hypothesis that cancer progresses in a multistep fashion, re- quiring activation of a number of oncogenes. Transgenic mice bearing distinct oncogenes are derived independently and then cross-mated to generate doubly transgenic animals. The synergistic behavior of ras and myc has been demonstrated in mammary gland differentiation and tumorigenesis. Cooperativity has also been established between ras, myc, and SV40 T-antigen in hepatocarcinogenesis. Peptide growth factors have been implicated in both physiologic and pathologic processes for many years, and are now being examined in the context of the whole animal. Transforming growth factor-a, which triggers cellular proliferation by binding to and activating the epidermal growth factor receptor, has been overexpressed in a wide variety of transgenic mouse organs, resulting in a constellation of phenotypic alterations, including metaplasia of the pancreas, neoplasia of the mammary gland and liver, abnormal organogenesis of the eye, and hyperplasia and psoriasis of the skin. Transgenic mice overexpressing nerve growth factor in pancreatic islet cells exhibit a striking increase in islet innervation by a single subtype of sympathetic neuron. Transgenic animals overproducing cytokines and hemopoietic growth factors have also been de- scribed: interleukin-4 causes an inflammatory disease reminiscent of an allergic reaction and perturbs $\mathrm{T}$ cell development; interleukin-5 induces eosinophilia and stimulates the production of autoantibodies; and granulocyte-macrophage colony stimulating factor stimulates the accumulation of macro- phages, inducing lesions of the eye and striated muscle, and premature death. Transgenic mice hold great potential as test systems for the improvement of the treatment of disease. A compelling argument for the value of transgenic animals as preclinical in vivo models can be made in the field of drug resistance [7]. A major obstacle to the treatment and eradication of human cancers is the ability of malignant cells to resist a broad spectrum of conventional chemotherapeutic agents. Cells can evade chemotherapy by overexpressing the cell-surface multidrug transporter P-glycoprotein, encoded by the multidrug resistance gene MDR1. Mice transgenic for human MDR1 have been created that express P-glycoprotein in their bone marrow, which becomes resistant to the leukopenia induced in non transgenic animals by chemotherapeutic drugs. Significantly, bone marrow resistance in MDR1 mice is reversed by simultaneous administration of known multidrug transporter inhibitors such as quinidine. These mice should prove valuable as a rapid test system to determine the efficacy of anti-cancer agents, especially those that reverse multidrug resistance in animals. Another major site of chemotherapeutic drug toxicity is the gastrointestinal tract. In a separate study, expression of a mutant dihydrofolate reductase transgene in the mouse intestine conveyed systemic resistance to methotrexate [6].

Recently the transgenic approach has provided new in sight into cardiovascular homeostasis and disease. Overexpression of the mouse ren- 2 transgene in the rat adrenal gland elicits severe hypertens showed that production of rat renin together with angiotensinogen causes elevated systolic blood pressure in transgenic mice, which can be reversed by the angiotensinconverting enzyme inhibitor captopril. In contrast, transgenic mice expressing the atrial natriuretic factor in the liver exhibit hypotension.

The role of oncogenes in tumorigenesis is being elucidated by targeting the expression of various types of oncogenes to specific tissues of transgenic animals. The dominant expression of activated oncogenes often results in the development of heritable tumors, clearly implicating these genes in onco- genesis. Several cogent and informative reviews have been written on this subject. I will focus on a few significant aspects of oncogenes in transgenic mice. 
Now the best-studied transgenic system for large-scale protein production is the mammary gland. The potential of the mammary gland as a bioreactor is being realized due to the cloning and characterization of genes encoding abundant milk proteins. Expression of genes of pharmaceutical interest can be targeted to the mammary gland by placing them under the control of the regulatory elements of genes encoding milk proteins such as the whey acidic protein, /3-lactoglobulin, and /3-casein. Pharmaceutically active proteins have been successfully expressed in mammalian milk, including human tissue plasminogen activator, ai-antitrypsin, coagulation factor IX, and interleukin 2, and could be used to treat human diseases such as myocardial infarction, emphysema, and hemophilia. Unfortunately, although expression of these transgenes has been accurately directed, the level of production remains variable and generally low. The incorporation of yet to be identified milk protein gene-specific LCRs into the transgene might insulate transgenes from the influence of inhibiting flanking sequences and stimulate their activity.

The genetic modification of animals faced active opposition from animal welfare and ethics point of views. The researchers working in this area should ensure the absence of welfare interference in using recombinant DNA technology and should applaud the merits of gene modification for protecting welfare of animals.

\section{Discussion and Conclusion}

Animal transgenesis is a technology which is promising in the future to change or replace the conventional use of drugs through the creation of disease-resistant animals and other methods of improving production and helps to avoid global food insecurity through diversification and improvement of agricultural products.

It is used to improve human health by filling organ gap. It will produce important pharmaceutical products to treat human disease. Animal Welfare and Ethics are the major issues which makes the acceptance of the technology controversial.

Transgenic animals are specifically designed to allow the study of how genes are regulated and how they affect the natural function of the body and development.

As transgenic animals are genetically engineered, they are also known as Genetically Modified Organisms (GMOs). The animals whose gene is manipulated is known as transgenic. These genes are passed on to the successive generations.

Transgenesis have a huge aspect in a wide variety of fields. It can provide animal models of human disease to help researcher to find new treatment and application.
It also makes the efficiency of transgenesis is low. The efficiency of transgenesis should be enhanced by the innovation of other efficient technique, the researchers of the technology should be well equipped and it should be of high level of ethical values and keeping the welfare of animals, innovation of new technologies should be invented for use in the field condition and awareness should be created.

\section{Bibliography}

1. Brinster R. "The effect of cells transferred into mouse blastocyst on subsequent development". Journal of Experimental Medicine (1974): 1049-1056.

2. Donnelly S., et al. "The Brave new World of Animal Biotechnology". Special Supplement, Hastings Center Report (1994).

3. Federation of European Laboratory Animal Science Associations (FELASA) September 1992, revised February 1995. Transgenic Animals - Derivation, Welfare, Use and Protection.

4. Gordon JW and Ruddle FH. "Integration and stable germ line transformation of genes injected into mouse pronuclei". Science 214 (1981): 1244-1246.

5. Gossler A., et al. "Transgenesis by means of blastocyst-derived embryonic stem cell line". Proceedings of the National Academy of Sciences of the United States of America 83 (1986): 90659069.

6. Jaenisch R. "Germ line integration and Mendelian transmission of the exogenous Moloney leukemia virus". Proceedings of the National Academy of Sciences of the United States of America 73 (1976): 1260-1264.

7. Moore CJ and Mepham TB. "Transgenesis and animal welfare". ATLA 23 (1995): 380-397.

\section{Assets from publication with us}

- Prompt Acknowledgement after receiving the article

- Thorough Double blinded peer review

- Rapid Publication

- Issue of Publication Certificate

- High visibility of your Published work

Website: www.actascientific.com/

Submit Article: www.actascientific.com/submission.php

Email us: editor@actascientific.com

Contact us: +919182824667 